\title{
Statistical Characteristics of Flow Field through Open and Semi-Closed Bileaflet Mechanical Heart Valve
}

\author{
Oleksandr Voskoboinyk ${ }^{\text {a }}$, Lidiia Tereshchenko ${ }^{{ }^{*}}$, Vladimir Voskoboinick ${ }^{\text {a }}$, \\ Gabriela Fernandez ${ }^{\mathrm{b}}$, Andrey Voskoboinick ${ }^{\mathrm{a}}$, Alberto Redaelli ${ }^{\mathrm{c}}$, \\ Gianfranco Beniamino Fiore ${ }^{c}$, Federico Lucherini ${ }^{c}$ \\ ${ }^{a}$ Institute of Hydromechanics of NAS of Ukraine, Kyiv, 03057, Ukraine. \\ ${ }^{b}$ Adjunct Faculty, Department of Geography, Center for Human Dynamics in the Mobile Age (HDMA), San Diego, California, United States. \\ ${ }^{c}$ Politecnico di Milano, Milan, Italy. \\ Received 02 August 2020; Accepted 26 October 2020
}

\begin{abstract}
The formation of thrombi on the streamlined surface of the bileaflet mechanical heart valves is one of the main disadvantages of such valves. Thrombi block the valve leaflets and disrupt the cardiovascular system. Diagnosis of thrombosis of the bileaflet mechanical heart valves is relevant and requires the creation of effective diagnostic tools. Hydroacoustic registration of the heart noise is one of the methods for diagnosing the operation of a mechanical heart valve. The purpose of the research is to determine the statistical characteristics of the vortex and jet flow through the open and semi-closed bileaflet mechanical heart valve, to identify hydroacoustic differences and diagnostic signs to determine the operating conditions of the valve. Experimental studies were conducted in laboratory conditions on a model of the left atrium and left ventricle of the heart between which there was the bileaflet mechanical heart valve. Hydrodynamic noise was recorded by miniature pressure sensors, which were located downstream of the valve. The vortex and jet flow behind the prosthetic heart valve were non-linear, random processes and were analyzed using the methods of mathematical statistics and probability theory. The integral and spectral characteristics of the pressure field were obtained and the differences in the noise levels and their spectral components near the central and side jets for the open and semi-closed mitral valve were established. It was shown that hydroacoustic measurements could be an effective basis for developing diagnostic equipment for monitoring the bileaflet mechanical heart valve operation.
\end{abstract}

Keywords: Bileaflet Prosthetic Mitral Valve; Jet Flow; Vortex Structures; Wall Pressure Fluctuations; Hydrodynamic Noise.

\section{Introduction}

The most important thing in human life is the smooth functioning of the cardiovascular system. Directed transfer of blood through arteries, veins, capillaries and other blood vessels, which is provided by the heart and valves, which are located between the atria and ventricles, allows a person to live. The heart is a biological muscle pump that supplies the body with the oxygenated blood and nutrients and takes away the blood saturated with carbon dioxide and waste metabolic products. The heart is divided by a partition into two cavities, which serve to collect the blood (left and right atria) and for muscle pumping of the blood (left and right ventricles). Between the atria and the ventricles are valves (mitral and tricuspid), which open when the ventricles are filled with the blood. From the ventricles, the blood through the aortic and pulmonary valves enters inside the aorta and pulmonary arteries, through which the blood enters into the lungs, where it is saturated with oxygen. These valves are opened when the ventricles are contracted and the mitral and

* Corresponding author: litere70@gmail.com

\section{doi) http://dx.doi.org/10.28991/SciMedJ-2020-0204-1}

$>$ This is an open access article under the CC-BY license (https://creativecommons.org/licenses/by/4.0/).

(C) Authors retain all copyrights. 
tricuspid valves are closed. Thus, the heart creates a unidirectional movement of the blood along the large (left side of the heart) and small (right side of the heart) circulation of the blood of the cardiovascular system and interferes with regurgitation or the reverse flow of the blood [1-4].

The cardiac cycle of the blood circulation in the human cardiovascular system consists of a diastole phase and a systole phase. When the left ventricle relaxes, the mitral valve (nature two-leaflets) is opened and the oxygenated blood in the lungs passes from the left atrium into the left ventricle through it. After the opening of the mitral valve, the blood velocity through the valve is increased and reaches its maximum in the diastole phase (wave E), and then it is decreased and flows with the small decreasing velocity (diastasis). Then the left atrium is contracted and in the blood flow again the velocity increase is observed (wave A). Typically, the maximum velocity in the wave $\mathrm{E}$ is higher than in the wave A. After this, the muscles of the left ventricle begin to contract (myocardial contraction) and the mitral valve is closed. When the pressure in the left ventricle exceeds the pressure in the aorta, then the aortic valve (nature three-leaflets) is opened. The blood flow in the systole phase is rushed inside the aorta and then inside other blood vessels of the circulatory system. Systole lasts about a third of the cardiac cycle. A similar cardiac cycle takes place in the small circulation of the blood of the cardiovascular system through the right atrium, the tricuspid valve, the right ventricle of the heart and the pulmonary valve [5-8].

Numerical and physical modeling of the features of the blood flow inside the cardiovascular system and the heart function has a long and rich history. Scientists all over the world pay considerable attention to this field of knowledge. This helps to reduce the number of diseases of the circulatory system and the entire cardiovascular system as a whole. New knowledge makes it possible to improve therapeutic and surgical methods of the treatment, to create effective artificial elements and designs of the replacement of damaged components of the cardiovascular system [1,9] Experimental studies of the blood movement and the features of the vortex and jet flow near the heart valves are a complex task for in vivo and in vitro studies [10-12]. This is due to the spatial and temporal limitations of the measuring instruments, the opacity of the blood flow and its non-Newtonian properties, the need not to disturb the flow and the complexity, and sometimes the impossibility of direct measurements inside the heart. In recent years, laser Doppler velocity meters, ultrasound and tomography complexes, microPIV technique and other measuring instruments have been used in experiments [13-17]. But they have their advantages and disadvantages. Many new scientific results were obtained by numerical simulations using various methods and techniques [18-24] that require experimental confirmation and verification. Therefore, an urgent task of modern scientific hemodynamic and biomechanic studies is conducting experimental studies with high-precision and miniature measuring instruments [2528]. These instruments must work in opaque non-Newtonian moving media and do not disturb the blood flow.

Heart diseases are one of the leading causes of death in people around the world. Pathologies of one or more valves lead to malfunction of the heart and the entire cardiovascular system. Most often, pathologies are recorded with the aortic valve, since it withstands the greatest pressure drops between the left ventricle and the main artery, which supplies the blood to the arterial system of the pulmonary circulation. Valve pathologies often require surgical treatment. More than 300,000 artificial heart valves are implanted annually in the world and among them about $70 \%$ are mechanical heart valves $[12,21]$. Bileaflet mechanical heart valves are the most common prosthetic valves. Typically, valve leaflets are made of pyrolytic carbon in the form of two lunate surfaces. The leaflets are attached to the annular surface of the valve base by means of hinge joints. Between the leaflets and the valve ring there is a technological gap of the order of 150 microns. It should be noted that this gap is one of the design flaws of the valve $[29,30]$.

Bileaflet mechanical heart valves have increased durability compared to bioprostheses [1, 17, 31], but are subject to hemolysis, platelet activity, and thrombosis, thromboembolism. Therefore, for patients with such valves, life-long anticoagulation therapy is necessary $[1,2,17,32,33]$. These defects are caused by the non-physiological dynamics of the blood flow through the valve, the rigidity of the valve leaflets, the formation of intense vortex and jet flows, and the increased shear stresses [34-36]. Intensive backflows are formed in the gaps of the hinged joints of the leaflets and the base of the valve, which destroy the red blood cells and platelet shells. Here thrombi are formed, which are attached to the valve leaflets in the area of stagnant flows near the hinged joints $[2,34,37,38]$. Cavitation areas with microbubbles in the blood flow are formed, during the closing of the leaflets of the mechanical valves due to a sharp decrease in pressure. Cavitation destroys the blood structure and elements and cause erosion of the streamlined surfaces [34, 39-41]. The formation of the thrombi on the surface of the leaflets of the bileaflet mechanical heart valve leads to serious interferences with the operation of the valve leaflets. Thrombi interfere with the opening of the leaflets or the closing of them, which leads to the regurgitation of the blood flow, the stenosis and the changes to the geodynamics of the cardiovascular system and metabolism.

This seriously disrupts the human cardiovascular system and leads to death when two leaflets of the heart valve will be closed. In order to prevent such a situation, it is necessary to diagnose the operation of mechanical valves and take the necessary measures to reduce the formation of thrombi by both therapeutic and surgical methods. In this regard, there is an urgent need to develop and create effective methods and tools for diagnosing heart valves. Among 
such methods it is offered to use hydroacoustic measurements of the noise for the operating conditions of the open and semi-closed bileaflet mechanical heart valve and on the basis of these measurements to reveal diagnostic signs that emphasize the urgency of carrying out such medical actions.

The purpose of this research is to determine the statistical characteristics of the vortex and jet flow through the open and semi-closed bileaflet mechanical heart valve, to identify hydroacoustic differences and diagnostic signs to determine the operating conditions of the valve.

\section{Materials and Methods}

When thrombi overlap the leaflet of the heart valves, their input sections are reduced. This leads to a significant increase in blood flow drag and increases flow velocity when fluid passes through the open valve leaflet. As a result, intense vortex and jet structures are separated from the valve leaflets, and the flow becomes unsteady and unstable with a high degree of turbulence. It is known that turbulent flows generate intense hydrodynamic noise, which has a sound and pseudo-sound nature [42-44]. Sound fluctuations of the velocity and pressure are associated with the compressibility of the medium, propagate into the environment with the sound velocity, which is determined by the elasticity of the medium, and obey the principle of superposition. Pseudo-sound fluctuations of the velocity and pressure are generated by vortex structures and jet flows, and these fluctuations are transferred with the velocity of movement of the vortex structures and jets. Pseudo-sound fluctuations in an unsteady turbulent flow are a nonlinear phenomenon and do not obey the principle of superposition. The intensity of the pseudo-sound fluctuations decays with distance from the source in proportion to the square of the distance.

Therefore, if to place the pressure fluctuation sensors near the jets that flow from the heart valve, they can record changes in hydrodynamic noise. This principle underlies the hydroacoustic diagnosis of the operation of the mitral valve. Naturally, pressure fluctuation sensors cannot be placed inside a person's heart. Therefore, heart noise sensors and accelerometers were located on the surface of the measuring bench in addition. Thus, hydrodynamic noises were recorded inside the model of the left ventricle, and noise and vibration fields were simultaneously recorded on the surface of the experimental bench. This makes it possible to determine the degree of transformation of hydrodynamic noise inside the bench into external noise and vibration on its surface. Differences in the intensity and spectral composition of the noise and vibration on the external surface of the heart model are the basis for creating a diagnostic complex of the operation conditions of the heart valve during thrombosis.

It is known that perturbations and instabilities in separating and jet flows, which are caused by the turbulence, are random processes $[45,46]$. Such processes are investigated using statistical analysis, which reflects the average and expected behavior of the characteristic properties and hydrodynamic parameters of such flows. In the formal representation of random processes, the vector components of velocity or vorticity, pressure and acceleration have a certain probability of accepting a specific value. A statistical description of such variables was carried out by obtaining integral and spectral characteristics. Four central statistical moments were determined in the studies, namely, the average values of the random variables, the probability density functions, variances and root-mean-square values of variables, skewness and kurtosis coefficients, and spectral characteristics of random variables. These functions and coefficients made it possible to determine the sources of pressure fluctuations and vibrations, their scales, directions and transfer velocities, intensities and spectral components [47, 48]. A comparison of the statistical characteristics of the pressure fields and vibrations in the operation conditions of the open and semi-closed valve made it possible to identify diagnostic signs and the possibility of hydroacoustic diagnosis of the formation of the thrombi on the leaflets of the mechanical heart valve.
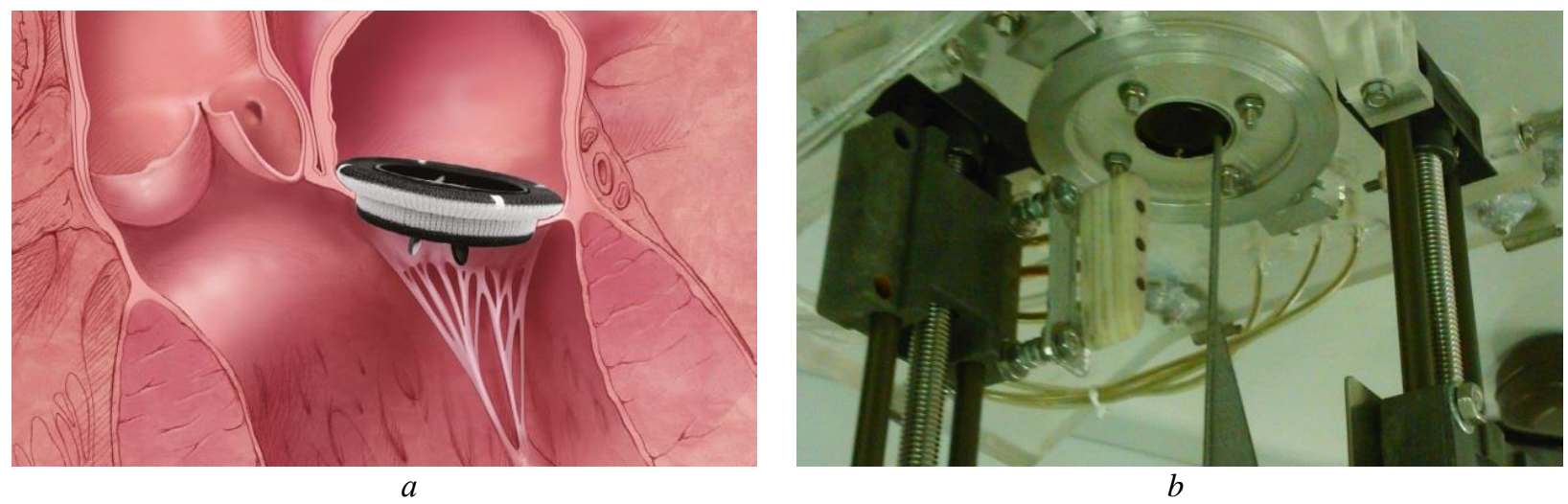

Figure 1. Bileaflet mechanical heart valve inside the left venticle (a) and the experimental bench (b) 


\section{Experimental Setup}

Physical simulation of the flow and overlap of the leaflets of mechanical heart valves by thrombi was performed in the laboratory at the Technical University "Politecnico di Milano" (Italy). A bileaflet mechanical heart valve (Figure 1) from Sorin Biomedica Cardio (Italy) with a diameter of $\mathrm{d}=25 \mathrm{~mm}$ was installed in the position of the mitral valve between the model of the left atrium and the model of the left ventricle [49, 50]. The studies were performed for the continual and pulsating flow of pure water and an aqueous solution of glycerol through an open and semi-closed valve. Behind the open bileaflet valve, the flow of fluid through the valve was divided into three jets: one narrow central jet between the leaflets and two "crescent" side jets between the leaflets and the annular base of the valve.

To solve this problem, miniature pressure, noise and acceleration sensors have been developed and manufactured. The pressure sensors were installed in a well-streamlined sensor's block, which consisted of five pressure fluctuation and absolute pressure sensors, which were mounted flush with the streamlined surface of this block and located at different distances downstream from the bileaflet valve (Figure 1b).

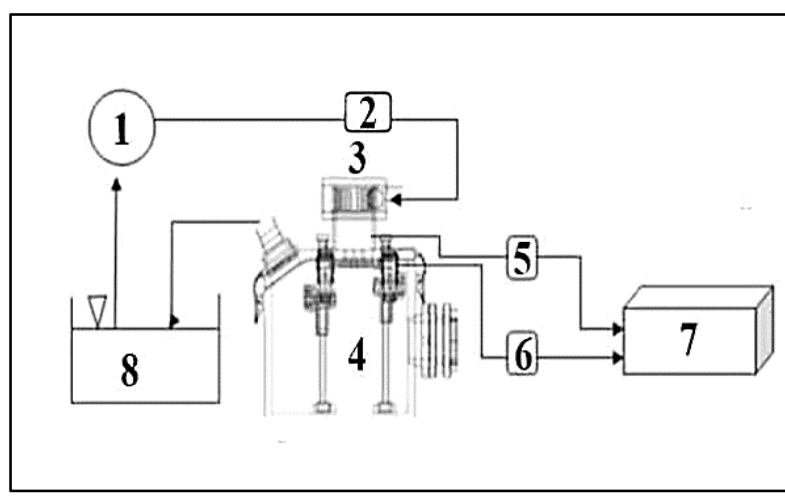

$a$

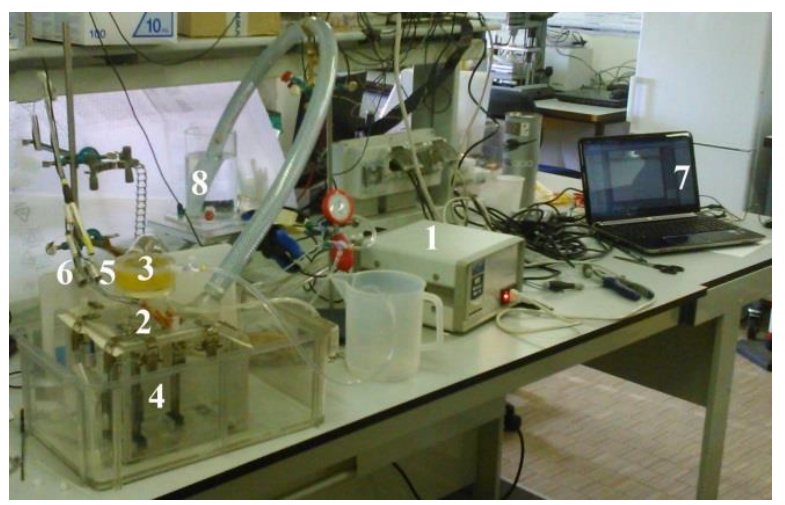

$b$

Figure 2. Schematic diagram (a) and photo (b) of the experimental stand

To conduct physical simulation of fluid flow through the bileaflet mechanical heart valve, an experimental stand was created [49-51], which is shown in Figure 2. The mitral valve was located in the opening between the model of the left atrium (3) and the model of the left ventricle (4). Pure water or an aqueous solution of glycerol from the settling chamber (8) by means of a pump (1) was fed into the atrial model and through the open or semi-closed valve entered the left ventricle model and then into the settling chamber. Sensors (5) and (6) recorded the field of pressure fluctuations in the lower flow of the valve, as well as noise and vibration on the surface of the experimental bench. An ultrasonic overhead velocity meter (2) was located on the inlet pipe to the bench and recorded the flow rate. The electrical signals of the sensors were fed to the recording equipment, data processing and analysis tools (7).

Pressure sensors, located in the sensor unit (Figure 3a), using a coordinate device (Figure 2b) moved along the studied jets, recording their hydrodynamic noise. Miniature wall pressure fluctuation sensors (Figure $3 b$ ) had the sensitive surface diameter of $1.3 \mathrm{~mm}$, and piezoresistive absolute pressure sensors had the sensitive surface diameter of $0.8 \mathrm{~mm}$. These sensors were mounted flush with the well streamlined sensor's block and did not disturb flow. Sensors recorded the pressure field in the near wake of the central jet or side jet, which flowed from the open or semiclosed mitral valve. Piezoceramic heart noise sensors and accelerometers were installed on the outer surface of the measuring bench, which is a model of the left atrium and left ventricle.

The electrical signals of the sensors were amplified, filtered and fed through a 16-channel analog-to-digital converter to a personal computer, where the appropriate programs and algorithms processed and analyzed the experimental dates using the apparatus of probability theory and mathematical statistics [52]. Simultaneous multipoint recording of pressures, noise and vibrations allowed investigating the space-time characteristics of hydroacoustic and vibration parameters, to identify sources of noise and vibration [48, 49, 53]. 


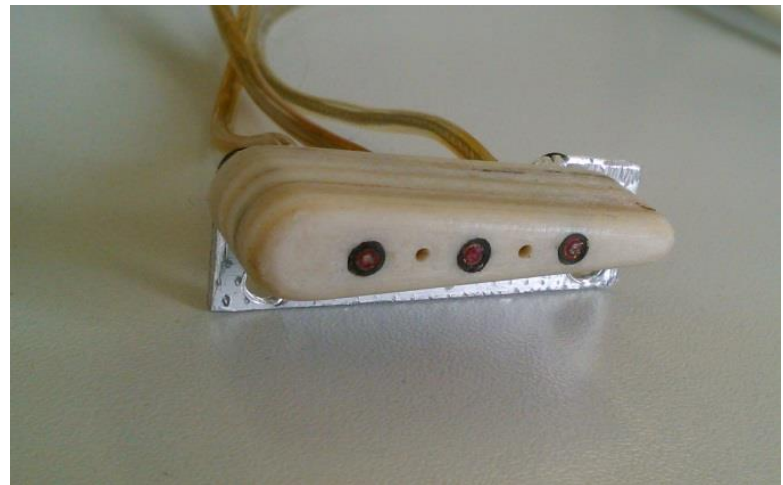

$a$

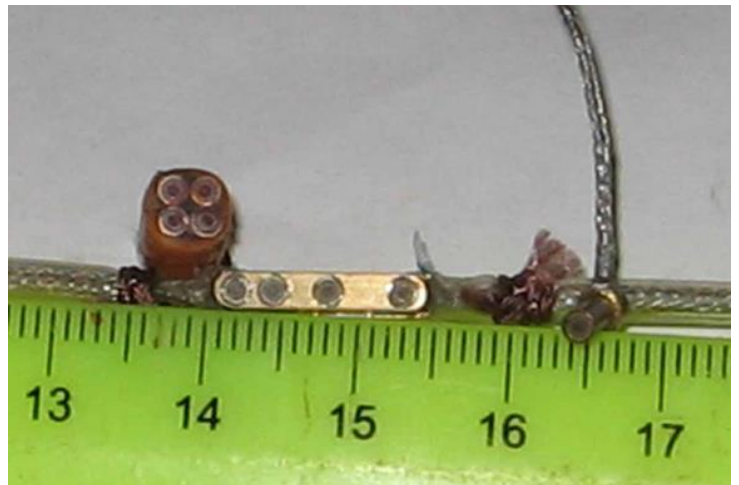

Figure 3. Sensor's block (a) and miniature pressure fluctuation sensors (b)

The measurement error of the integral values of the velocity and pressure fields, and also vibration did not exceed $10 \%$ (95\% reliability). The measurement error of the flow rate is no more than $3 \%$. The measurement error of the spectral characteristics of the velocity, pressure fluctuations and accelerations is no more than $2 \mathrm{~dB}$ in the frequency range from $0.01 \mathrm{~Hz}$ to $2 \mathrm{kHz}$ with a confidence probability of 0.95 or $2 \sigma$.

\section{Results and Discussion}

Features of jet and vortex flow downstream from the open and semi-closed bileaflet mechanical mitral valve were studied in the steady flow of the pure water and the aqueous solution of glycerol flow rate from $51 / \mathrm{min}$ to $201 / \mathrm{min}$. Such flow rates correspond to those observed during the diastole of the cardiac cycle of the valve. Piezoresistive pressure sensors measured the total pressure, which consisted of averaged and fluctuating components, in the form $p=P+P^{\prime}$. Mean pressure or mathematical expectation was determined by the dependence:

$\mu(p)=\int_{-\infty}^{\infty} p P(p) d p=\bar{P}$

Where $P^{\prime}$ are the oscillations or fluctuations of the pressure on the streamlined surface, $P(p)$ is the probability of the pressure that satisfies the condition:

$$
\int_{-\infty}^{\infty} P(p) d p \equiv 1
$$

The root mean square value of pressure fluctuations was determined by the dependence:

$$
p_{r m s}^{\prime}=\sqrt{\overline{\left(p^{\prime}\right)^{2}}}=\sigma_{p}^{1 / 2}
$$

Where $\sigma_{p}$ is the dispersion of the pressure fluctuation field.

Mean values and root mean square values of the pressure inside the model of the left ventricle of the heart and in the model of the left atrium are shown in Figure 4. Here Figure 4a presents the results of measurements the mean pressure, which is calculated according to dependence (1), for the flow of the pure water through the open and semiclosed valve. Curve 1 is measured near the side jet and curve 2 near the central jet of the open valve, and curves 3 and 4 are measured near, respectively, the side and central jet, but already semi-closed valve. Curve 5 is measured inside the atrium when the valve is fully open, and curve 6 is measured inside the atrium for a semi-closed valve. Figure $4 \mathrm{~b}$ presents the results of measurements the root mean square values of the pressure fluctuations, which are calculated according to dependence (2), also for the flow of the pure water through the open and semi-closed valve. Curves in Figure $4 \mathrm{~b}$ correspond to those presented in Figure 4a. The results show that with increasing flow rate, the mean pressure and root mean square pressure fluctuations near the side jet and inside the atrium for operating conditions of the semi-closed valve is higher than for the open valve. 

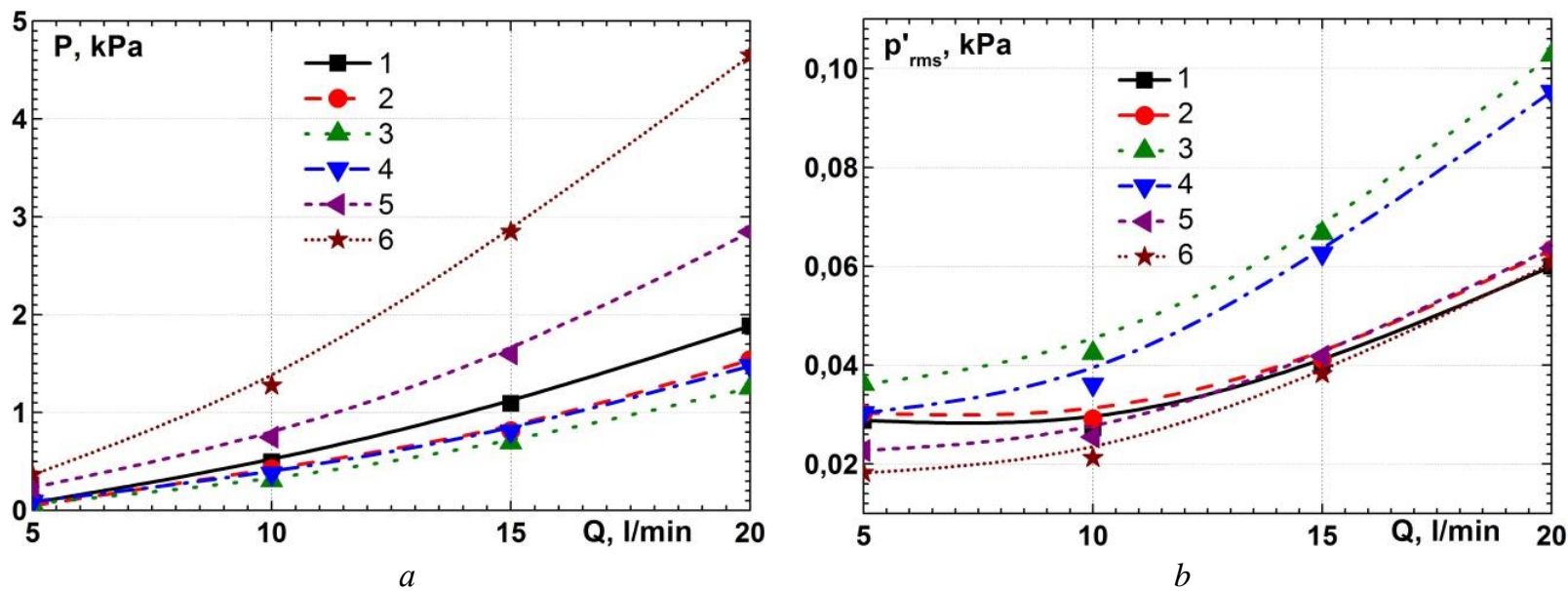

Figure 4. Integral characteristics of the pressure field: mean values of the pressure (a) and root mean square values of the pressure fluctuations (b)

It is known [52] that the characteristic measure of the random variable (pressure fluctuations) is the probability $P\left(p^{\prime}\right)$ that the random variable $\left(p^{\prime}\right)$ will take one value or another or falls into a given interval $\left(\Delta p^{\prime}\right)$. The first derivative of the distribution function of the random variable $p\left(p^{\prime}\right)$ sets the velocity of change of the probability depending on the value of the pressure fluctuations. Usually, the function $p\left(p^{\prime}\right)$ is estimated by calculating the probability that the instantaneous value of an individual implementation is in a narrow interval and is divided by the width of the interval according to the dependence:

$\rho\left(p^{\prime}\right)=\lim _{\Delta p^{\prime} \rightarrow 0} \frac{P\left(p^{\prime}+\Delta p^{\prime}\right)-P\left(p^{\prime}\right)}{\Delta p^{\prime}}=\partial P\left(p^{\prime}\right) / \partial p^{\prime}$

The total area under the probability density graph is equal to unity, which indicates the reliability of the event. Therefore, the probability density is one of the main functions of describing the probable structure of the random process. It is also used to determine the normality of the process, identify non-linearity and analyze extreme values.
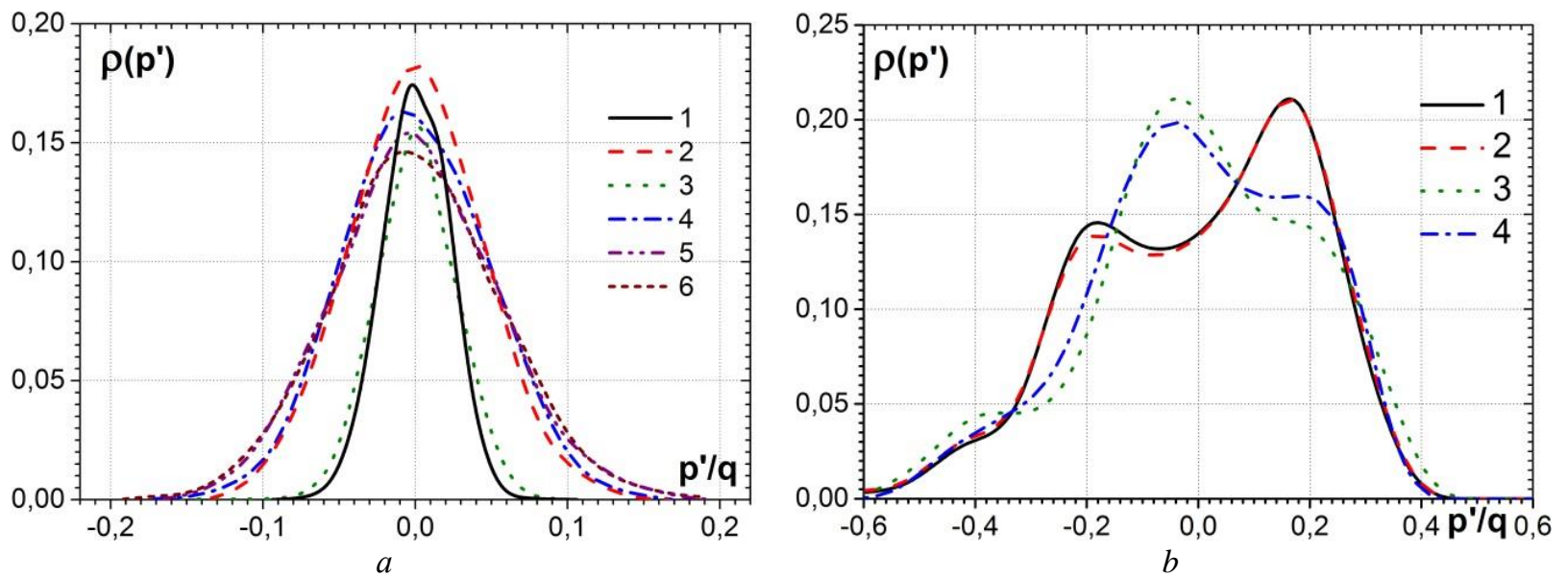

Figure 5. Probability density functions of the pressure fluctuations: stationary (a) and pulsating fluid flow (b)

The probability density functions of the pressure fluctuations near the side jet of the open and semi-closed valve, through which the steady and pulsating stream flows, are calculated according to dependence (3) and shown in Figure 5. Curve 1 in Figure 5a presents the results of measurements of the pressure fluctuations near the side jet of the pure water flow with flow rate of $15 \mathrm{l} / \mathrm{min}$ through the open valve. Curve 2 is obtained for the same research conditions, but for the semi-closed valve. Curves 3-6 were measured for the steady flow of the glycerol solution of different concentrations. Thus, curve 3 was measured near the side jet of the open valve, through which flowed the solution of $43 \%$ glycerol and $57 \%$ water. Curve 4 was measured for the semi-closed valve and $35 \%$ glycerol in the aqueous solution, curve 5 - 38\% glycerol and curve $6-43 \%$ glycerol. Figure $5 \mathrm{~b}$ shows the results of measurements of the hydrodynamic noise near the side jet of the pulsating flow of the pure water with a frequency $(f)$ of $1 \mathrm{~Hz}$ or 60 beats per minute. Curves 1, 2 were measured for the open valve conditions, and curves 3, 4 were measured for the semi-closed valve conditions. Curve 1 was measured at the distance equal to the diameter $(d)$ of the valve, curve 2 was measured at the distance $x=1.2 d$, curve 3 was measured at the distance $x=d$, and curve 4 was measured at the 
distance $x=1.2 d$. It should be noted that the dependencies in Figure 5a are characteristic of random processes ("bellshaped" shape of the curve), and the dependencies in Figure $5 \mathrm{~b}$ represent a set of the random process with the oscillatory determinate process $[10,12]$. This is not surprising, because in the pulsating flow through the open or semiclosed valve, harmonic oscillations with cardiac rhythm are superimposed on the field of stochastic pressure fluctuations of the jet and vortex flow. The pressure fluctuations near the side jet of the open valve, regardless of the viscosity of the fluid, have a low probability of high-amplitude pressure fluctuations. With increasing viscosity of the glycerol solution, the pressure fluctuations of small amplitude near the side jet of the steady flow through the semiclosed valve are significantly damped, which is clearly illustrated in Figure 5a.

The central statistical moments, the skewness coefficient (third moment) was determined:

$$
S k=\overline{\left(p^{\prime}\right)^{3}} /\left(\sigma_{p}\right)^{3 / 2}
$$

And the kurtosis coefficient (fourth central moment) was determined by the dependence:

$$
\operatorname{Kur}=\overline{\left(p^{\prime}\right)^{4}} /\left(\sigma_{p}\right)^{2}-3
$$

Changes in statistical moments of higher orders, namely, the coefficients of the skewness and kurtosis, which are calculated according to dependences (4 and 5), are shown in Figure 6. Hydrodynamic noise was measured in the near wake of the valve about the side jet for the steady flow of the pure water and the aqueous glycerol solution. Here, curves 1-4 are measured for open valve conditions, and curves 5-8 are measured for semi-closed valve conditions. Curves 1 and 5 were obtained for the pure water flow, curves 2 and 6 - for the solution of $35 \%$ glycerol, curves 3 and 7 - for the solution of $38 \%$ glycerol and curves 4 and 8 - for the solution of $43 \%$ glycerol. Thus, for the low flow rate in the pressure fluctuation field, positive pressure fluctuation values prevail, and with increasing flow rate, the probability of negative pressure fluctuations increases, especially for the pure water flow (Figure 6a). The kurtosis coefficient of the pressure fluctuations for the conditions of the pure water flow and the non-Newtonian fluid with the high viscosity differ from each other (Figure 6b). Thus, for low flow rates in the field of the pressure fluctuations of the pure water flow, the pressure fluctuations of the large amplitude predominate, and with increasing water flow rate, small-scale pressure fluctuations begin to prevail. The opposite trend is observed for the flow of the aqueous solution of glycerine, especially when its concentration is increased.
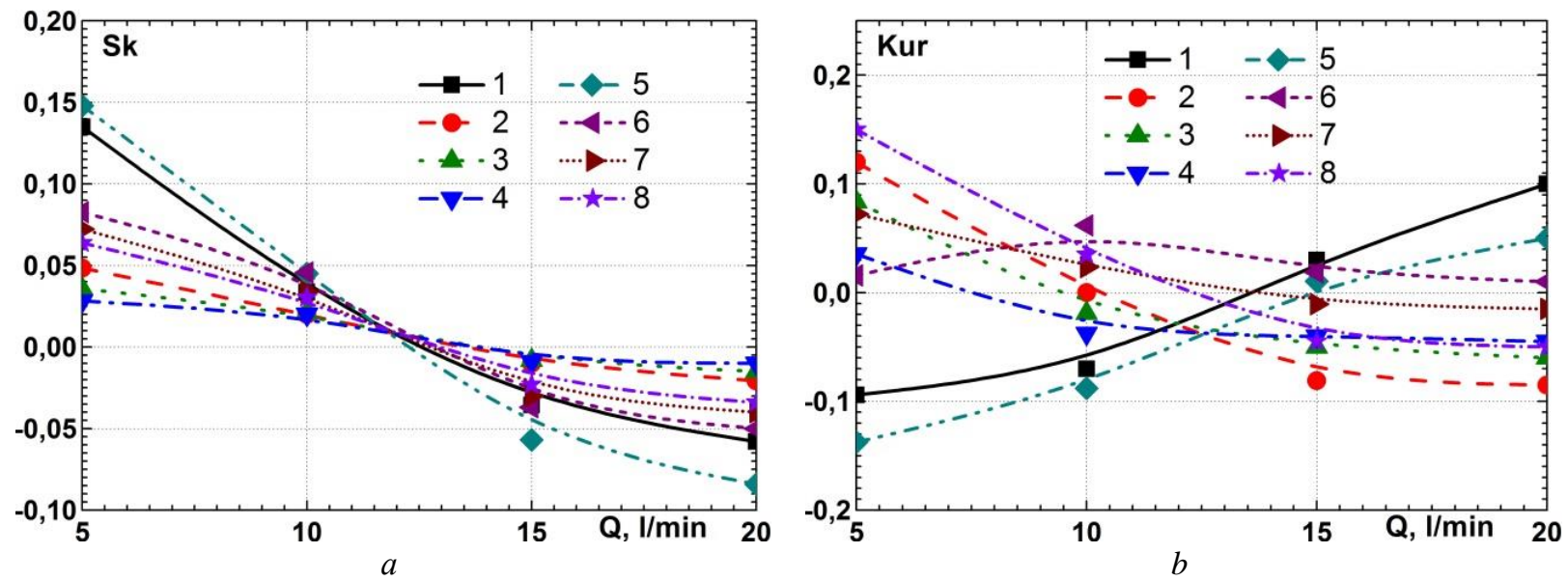

Figure 6. Coefficients of the skewness (a) and kurtosis (b) of the field of pressure fluctuations about the side jet in the near wake of the bileaflet mechanical mitral heart valve

Spectral analysis of the experimental data made it possible to determine the power spectral density of the pressure fluctuations from the dependence:

$P(\omega)=\frac{1}{2 \pi} \int_{-\infty}^{\infty} R_{p^{\prime}}(\tau) \exp (-i \omega \tau) d \tau$

Where $\omega=2 \pi f$ is the circular frequency, $\tau$ is the delay time, and $R_{p^{\prime}}(\tau)$ is the autocorrelation of the pressure fluctuations, which was determined from the dependence:

$$
R_{p^{\prime}}(\tau)=\overline{p^{\prime}(\tau) \cdot p^{\prime}(t+\tau)}
$$



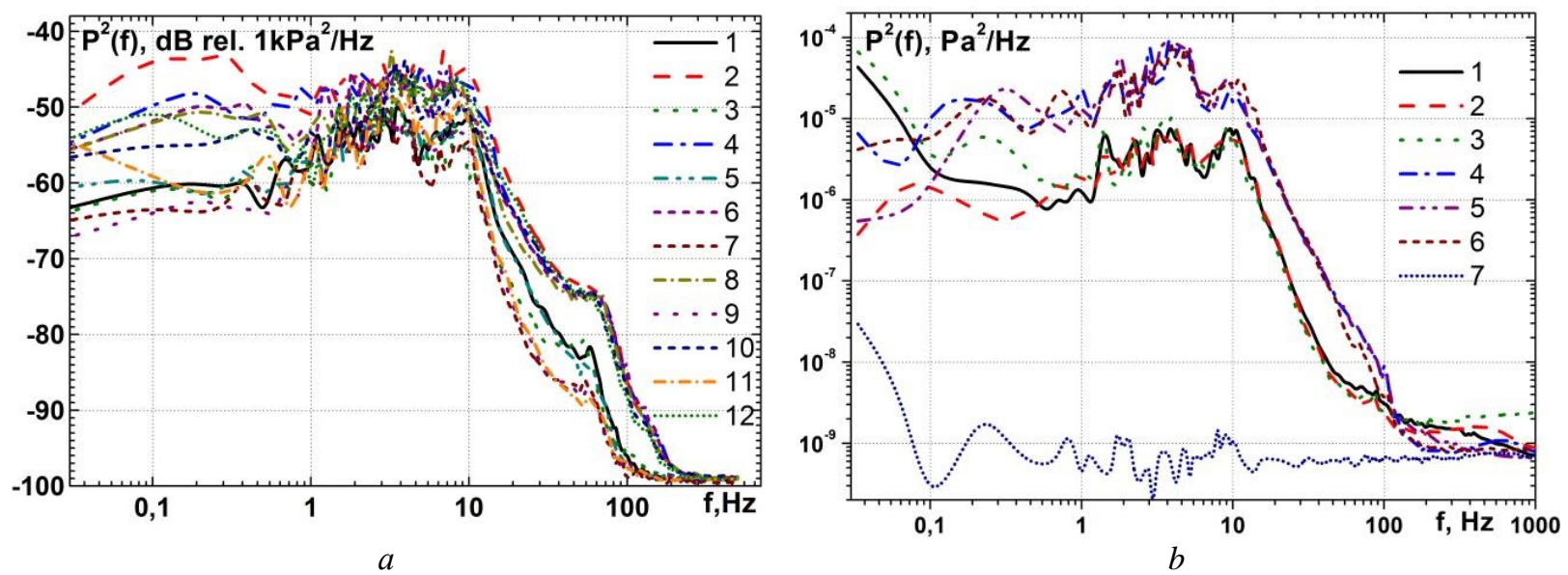

Figure 7. Power spectral densities of the pressure fluctuations near the side jet for the pure water flow (a) and for the aqueous glycerol solution flow (b) through the open and semi-closed bileaflet heart valve

The power spectral densities of the pressure fluctuations calculated according to Equation 6 are presented in Figure 7 for different conditions of the research. Measurements were performed near the side jet of the steady flow of the pure water and glycerol solutions of different concentrations through the open and semi-closed bileaflet heart valve. Figure 7 a shows the spectra of the pressure fluctuations measured along the side jet at different distances from the valve for the flow of the pure water with the flow rate of $151 / \mathrm{min}$. Curves 1-6 are measured for the open valve and curves 7-12 are measured for the semi-closed valve. Curves 1 and 7 are registered at the distance $x=d$ from the valve, curves 2 and 8 - at the distance $x=1.1 d$, curves 3 and $9-x=1.2 d$, curves 4 and $10-x=1.4 d$, curves 5 and $11-x=2.4 d$ and curves 6 and $12-x=3 d$. The results of the research show that with the distance from the valve the spectral levels of the pressure fluctuations decrease for the operating conditions of the open valve, and an insignificant decrease is for the semi-closed valve, especially in the high-frequency region. The intensity of the pressure fluctuations near the side jet for the semi-closed valve is (3-4) times higher than for the open valve.
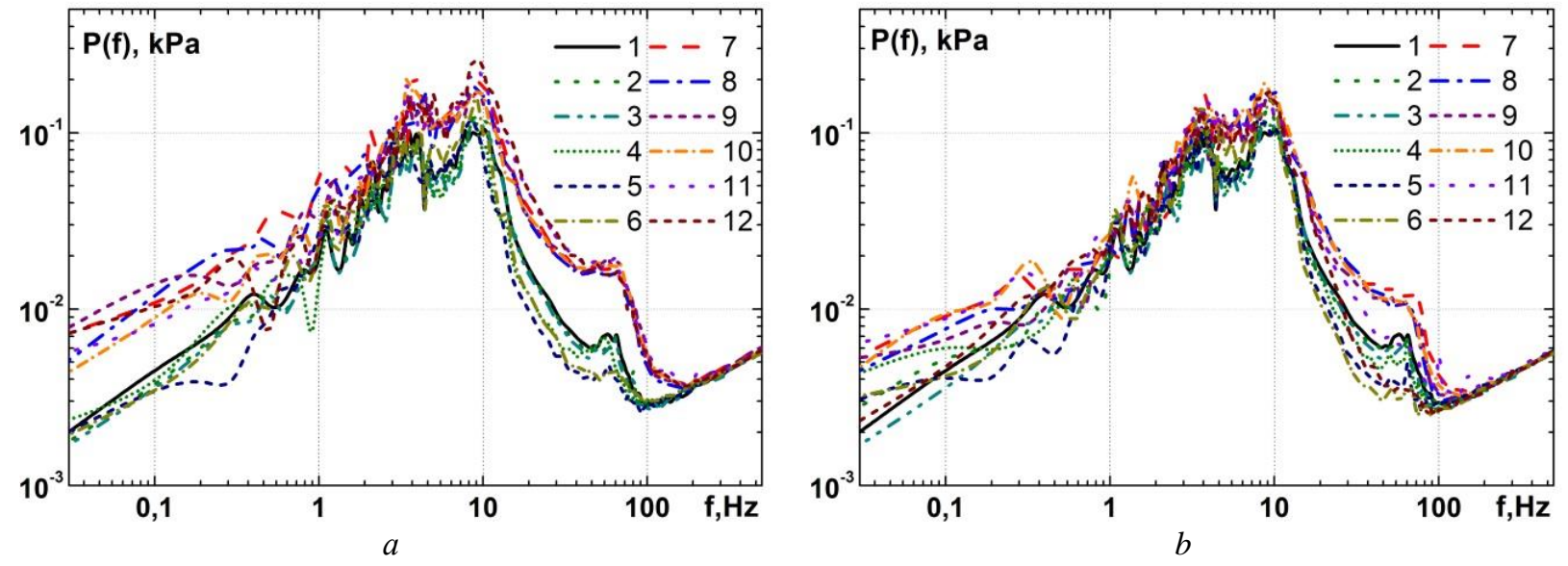

Figure 8. Pre-multiplied power spectral densities of the pressure fluctuations near the side jet (a) and near the central jet (b) for the pure water flow through the open and semi-closed bileaflet heart valve

The power spectral densities of the pressure fluctuations near the side jet of the aqueous solution of glycerol (43\% glycerol and 57\% water) through the open and semi-closed valve are presented in Figure 7b. Here, curves 1-3 are measured for the open valve, curves 4-6 are measured for the semi-closed valve, and curve 7 is an ambient noise. Curves 1 and 4 are obtained in the near wake of the valve at the distance $x=d$ from the valve, curves 2 and 5 - $x$ $=1.1 d$ and curves 3 and $6-x=1.2 d$. Here, as in Figure 7a, there is a significant difference in the spectra of the pressure fluctuations for the semi-closed and open valve, which is proposed to be used in the diagnosis of thrombosis on the streamlined surface of the heart valve. The results showed that the intensity of the pressure fluctuations near the side jet in the near wake of the semi-closed valve is higher than the open valve.

The pre-multiplied power spectral densities of the pressure fluctuations in the form $P(f)=\left(f P^{2}(f)\right)^{1 / 2}$ are presented in Figure 8 for the pure water flow through the open and semi-closed mitral valve with the flow rate of $15 \mathrm{l} / \mathrm{min}$. Such a representation of the spectral dependences makes it possible to show the frequency components of the pressure fluctuation levels in the near wake of the bileaflet mechanical heart valve. Figure $8 \mathrm{a}$ presents the measurement results of the pre-multiplied spectra of the pressure fluctuations near the side jet, and Figure $8 \mathrm{~b}$ shows 
the pre-multiplied spectra near the central jet in the near wake of the valve. Curves 1-6 are measured for the open valve, and curves 7-12 are measured for the semi-closed valve. Curves 1 and 7 are registered at the distance $x=d$ from the valve, curves 2 and 8 - at the distance $x=1.1 d$, curves 3 and $9-x=1.2 d$, curves 4 and $10-x=1.4 d$, curves 5 and $11-x=2.4 d$ and curves 6 and $12-x=3 d$. The research results show that the highest levels of the pressure fluctuations in the near wake of the valve are observed in the frequency range (5-12) Hz. This frequency range of the hydrodynamic noise is due to the features of the vortex and jet flow, which is formed downstream from the open or semi-closed valve. In the frequency range from $30 \mathrm{~Hz}$ to $100 \mathrm{~Hz}$, there is a significant difference between the hydrodynamic noise levels for the operating conditions of the open and semi-closed mitral valve.
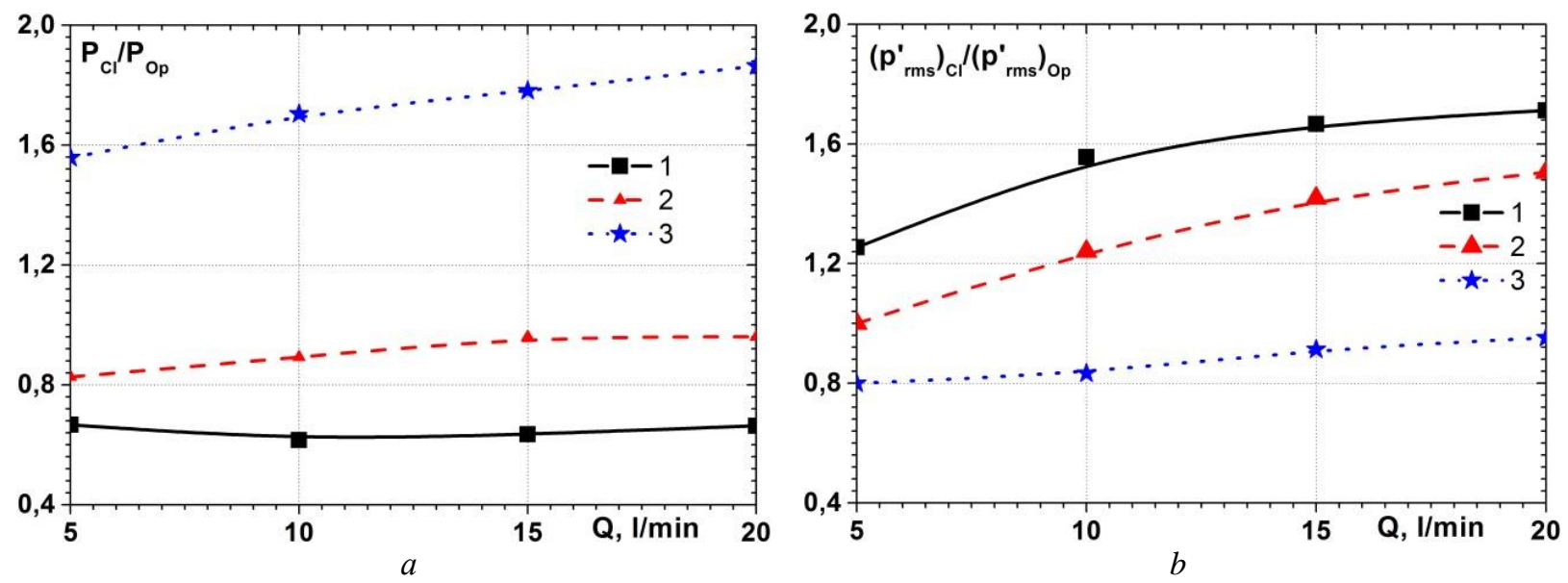

Figure 9. Ratios of the mean pressures (a) and the root-mean-square values of the pressure fluctuations (b) of the hydrodynamic noise inside the left ventricle model of the heart and the atrium model

The ratios of the mean pressures and the root-mean-square values of the pressure fluctuations of the hydrodynamic noise near the side and central jets in the near wake of the mitral valve inside the model of the left ventricle of the heart and inside the model of the atrium in front of the mitral valve under the operating conditions of the semi-closed valve to the open valve are shown in Figure 9. Figure 9a shows the ratios of the mean pressure values of the semiclosed valve to the open valve, and Figure $9 \mathrm{~b}$ shows the ratios of the rms values of the pressure fluctuations. Curve 1 is measured near the lateral jet, curve 2 is measured near the central jet inside the left ventricular model, and curve 3 is measured in front of the valve inside the atrium model. It was found that the mean pressures inside the left ventricular model are slightly decreased under the conditions of the semi-closed valve and significantly increased inside the left atrium model with an increase in the flow rate of the pure water (Figure 9a). The rms values of the pressure fluctuations in the common frequency band are increased under the conditions of the semi-closed valve and, with an increase in the pure water flow rate, the ratios become larger, especially near the side jet (Figure 9b).
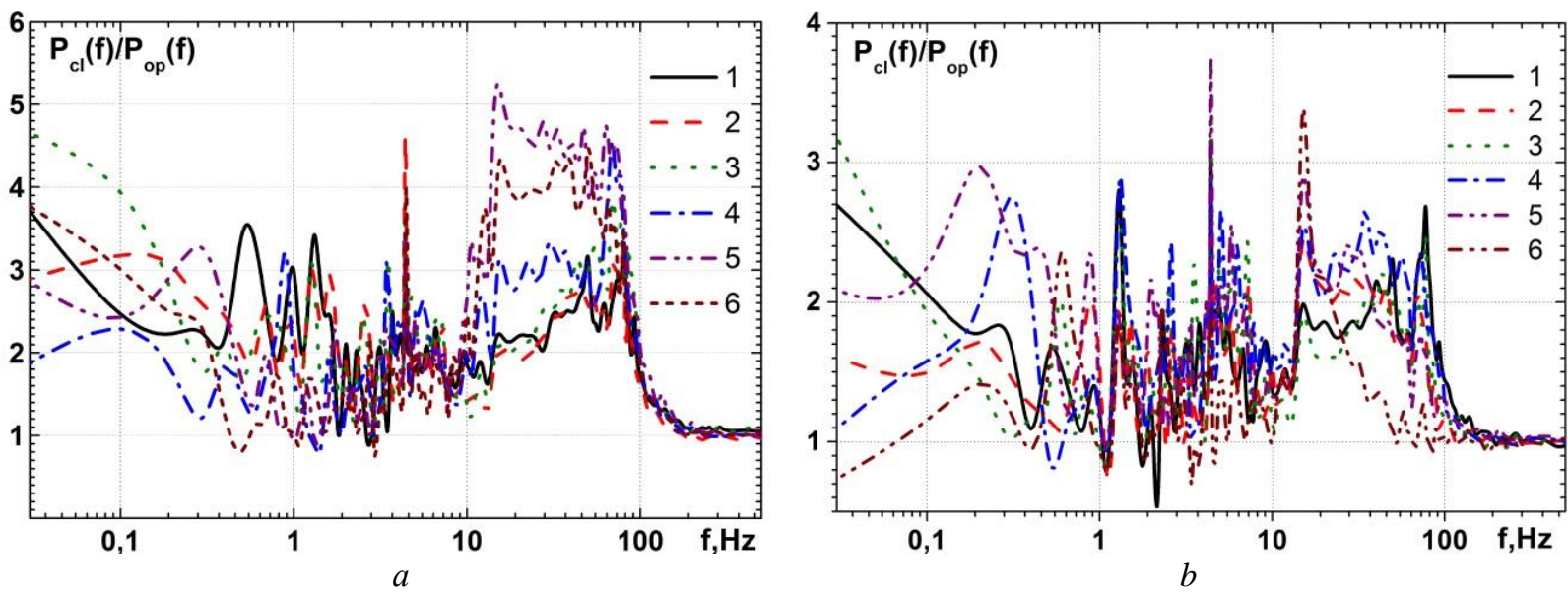

Figure 10. Ratios of the power spectral densities of the pressure fluctuations through the semi-closed valve to the open valve near the side jet (a) and near the central jet (b)

Figure 10 shows the ratio of the levels of the spectral components of the pressure fluctuations, which are measured for the pure water flow of $15 \mathrm{l} / \mathrm{min}$ through the semi-closed valve to those measured through the open valve. Figure 10a shows the ratio of spectral levels near the side jet, and Figure 10b - near the central jet. Measurements were made along these jets and curve 1 is measured at the distance from the valve $x=d$, curve 2 is measured at the 
distance $x=1.1 d$, curve $3-x=1.2 d$, curve $4-x=1.4 d$, curve $5-x=2.2 d$ and curve $6-x=3 d$. The results of the research showed that with the distance from the valve the difference of spectral levels in the hydrodynamic noise of the semi-closed valve and the open valve gradually decreases. The difference in spectra remains larger near the side jet and remains (2-3) times higher in the frequency range from $0.1 \mathrm{~Hz}$ to almost $100 \mathrm{~Hz}$. It should also be noted that the hydrodynamic noise near the side jet of the semi-closed valve is higher (4-5) times in the frequency range (3080) $\mathrm{Hz}$ than near the same side jet, but the open valve (Figure 10a). Such a large difference in spectral levels in this frequency range can be used in hydroacoustic diagnostics of the operation of the bileaflet mechanical heart valve.

Thus, based on a statistical analysis of the results of experimental studies in laboratory conditions of the operation of the bileaflet mechanical heart valve at the position of the mitral valve, the hydroacoustic characteristics of the vortex and jet flow through the valve were obtained. The features of the hydrodynamic noise in the near wake of the valve were studied when modelling the conditions of thrombi on one of the valve leaflets. The integral and spectral characteristics of the pressure field were obtained inside the model of the left ventricle of the heart and in the atrium. The changes in the statistical moments of the pressure fluctuations depending on the operating conditions of the mitral valve for the flow of the pure water and the glycerol solution of the steady and pulsating flow are shown. The levels of the spectral components of the pressure fluctuation field were determined depending on the distance to the valve for various flow rates through the open and semi-closed valve. Differences in the levels of the hydrodynamic noise in the near wake of the open and semi-closed mitral valve were revealed. It is indicated that hydroacoustic diagnostics, especially in certain frequency ranges, can be an effective means of determining thrombosis on the bileaflet mechanical heart valve.

\section{Conclusion}

The results of the research showed that the statistical analysis of the experimental data of the researches of the hydroacoustic characteristics of the jet and vortex flow downstream from the bileaflet mechanical heart valve is an effective tool in diagnosing the operating conditions of the valve. It was found that the integral characteristics and especially the spectral characteristics of the pressure fields had significant differences in the operating conditions of the semi-closed valve (simulation of the action of thrombi on the valve leaflets) and the open valve. It was established that the pressure fluctuations near the side jet of the open valve, regardless of the viscosity of the fluid, have a low probability of high-amplitude pressure fluctuations. With increasing viscosity of the glycerol solution, the pressure fluctuations of small amplitude near the side jet of the steady flow through the semi-closed valve are significantly damped. The pressure fluctuations of the large amplitude predominate for low flow rates in the field of the pressure fluctuations in the near wake of the valve for the pure water flow and small-scale pressure fluctuations begin to prevail with increasing water flow rate.

Differences in hydrodynamic characteristics of the steady and pulsating flow through the open and semi-closed valve are revealed. The intensity of the pressure fluctuations near the side jet for the semi-closed valve is (3-4) times higher than for the open valve. It was found that the hydrodynamic noise of the semi-closed valve is several times higher than that noise of the open valve, which is especially evident in different frequency ranges of the pressure fluctuation spectrum depending on the operating conditions of the bileaflet mechanical heart valve. The research results show that the highest levels of the pressure fluctuations in the near wake of the valve are observed in the frequency range (5-12) Hz. This frequency range of the hydrodynamic noise is due to the features of the vortex and jet flow, which is formed downstream from the open or semi-closed valve. In the frequency range from $30 \mathrm{~Hz}$ to $100 \mathrm{~Hz}$, there is a significant difference between the hydrodynamic noise levels for the operating conditions of the open and semi-closed mitral valve. The hydrodynamic noise near the side jet of the semi-closed valve is higher (4-5) times in the frequency range $(30-80) \mathrm{Hz}$ than near the same side jet, but the open valve.

\section{Data Availability}

All data included in this study are available upon request by contact with the corresponding authors.

\section{Authors' Contributions}

Oleksandr Voskoboinyk and Vladimir Voskoboinick conceived of the idea and helped write the manuscript. Oleksandr Voskoboinyk, Vladimir Voskoboinick and Lidiia Tereshchenko conducted experimental studies. Vladimir Voskoboinick, Victor Tkachenko and Andrey Voskoboinick conducted processing and analysis of experimental data. Vladimir Voskoboinick and Lidiia Tereshchenko wrote the main manuscript text. Oleksandr Voskoboinyk, Igor Kudybin and Andrey Voskoboinick contributed to interpretation of the results and prepared the figures. All authors reviewed and edited the manuscript. All authors listed in the manuscript have concurred with the content of the manuscript and submission. 


\section{Funding Statement}

This work was supported by the EU-financed project EUMLS (EU-Ukrainian Mathematicians for Life Sciences) grant agreement PIRSES-GA-2011-295164-EUMLS and AMMODIT (Approximation Methods for Molecular Modelling and Diagnosis Tools) - project reference: 645672, funded under: H2020-EU.1.3.3 - under the MSCARISE-2014 (Marie Sklodowska-Curie Research and Innovation Staff) of Program Horizon 2020.

\section{Acknowledgments}

The authors are grateful for the help to professors Jürgen Prestin and Alberto Redaelli for leading and coordinating research on the EUMLS and AMMODIT projects, to Professor Gianfranco Beniamino Fiore, Assistant Professor Riccardo Vismara and researcher Federico Lucherini for their active participation in the experimental work.

\section{Declaration of Competing Interest}

The authors declare that they have no known competing financial interests or personal relationships that could have appeared to influence the work reported in this paper.

\section{Ethical Approval}

The manuscript does not contain experiments on animals and humans; hence ethical permission not required.

\section{References}

[1] Sotiropoulos, F., Le, T. B., \& Gilmanov, A. (2016). Fluid Mechanics of Heart Valves and Their Replacements. Annual Review of Fluid Mechanics, 48(1), 259-283. doi:10.1146/annurev-fluid-122414-034314.

[2] Yoganathan, A. P., He, Z., \& Casey Jones, S. (2004). Fluid Mechanics of Heart Valves. Annual Review of Biomedical Engineering, 6(1), 331-362. doi:10.1146/annurev.bioeng.6.040803.140111.

[3] Zakaria, M. S., Ismail, F., Tamagawa, M., Aziz, A. F. A., Wiriadidjaja, S., Basri, A. A., \& Ahmad, K. A. (2017). Review of numerical methods for simulation of mechanical heart valves and the potential for blood clotting. Medical \& Biological Engineering \& Computing, 55(9), 1519-1548. doi:10.1007/s11517-017-1688-9.

[4] Reul, H., Talukder, N., \& Mu“ller, E. W. (1981). Fluid mechanics of the natural mitral valve. Journal of Biomechanics, 14(5), 361-372. doi:10.1016/0021-9290(81)90046-4.

[5] Sacks, M. S., David Merryman, W., \& Schmidt, D. E. (2009). On the biomechanics of heart valve function. Journal of Biomechanics, 42(12), 1804-1824. doi:10.1016/j.jbiomech.2009.05.015.

[6] Barker, A. J., \& Markl, M. (2011). Editorial. European Journal of Cardio-Thoracic Surgery, 39(6), 805-806. doi:10.1016/j.ejcts.2011.01.006.

[7] Votta, E., Le, T. B., Stevanella, M., Fusini, L., Caiani, E. G., Redaelli, A., \& Sotiropoulos, F. (2013). Toward patient-specific simulations of cardiac valves: State-of-the-art and future directions. Journal of Biomechanics, 46(2), $217-228$. doi:10.1016/j.jbiomech.2012.10.026.

[8] Mittal, R. (2018). Matters of the heart. Journal of Fluid Mechanics, 844, 1-4. doi:10.1017/jfm.2018.229.

[9] Kheradvar, A., Groves, E. M., Simmons, C. A., Griffith, B., Alavi, S. H., Tranquillo, R., ... Little, S. H. (2014). Emerging Trends in Heart Valve Engineering: Part III. Novel Technologies for Mitral Valve Repair and Replacement. Annals of Biomedical Engineering, 43(4), 858-870. doi:10.1007/s10439-014-1129-y.

[10] Vismara, R., Pavesi, A., Votta, E., Taramasso, M., Maisano, F., \& Fiore, G. B. (2011). A Pulsatile Simulator for thein Vitro Analysis of the Mitral Valve with Tri-Axial Papillary Muscle Displacement. The International Journal of Artificial Organs, 34(4), 383-391. doi:10.5301/ijao.2011.7729.

[11] Vismara, R., Leopaldi, A. M., Piola, M., Asselta, C., Lemma, M., Antona, C., ... Fiore, G. B. (2016). In vitro assessment of mitral valve function in cyclically pressurized porcine hearts. Medical Engineering \& Physics, 38(4), $346-353$. doi:10.1016/j.medengphy.2016.01.007.

[12] Meschini, V., de Tullio, M. D., Querzoli, G., \& Verzicco, R. (2017). Flow structure in healthy and pathological left ventricles with natural and prosthetic mitral valves. Journal of Fluid Mechanics, 834, 271-307. doi:10.1017/jfm.2017.725.

[13] Dasi, L. P., Ge, L., Simon, H. A., Sotiropoulos, F., \& Yoganathan, A. P. (2007). Vorticity dynamics of a bileaflet mechanical heart valve in an axisymmetric aorta. Physics of Fluids, 19(6), 067105. doi:10.1063/1.2743261.

[14] Bellofiore, A., Donohue, E. M., \& Quinlan, N. J. (2011). Scale-up of an unsteady flow field for enhanced spatial and temporal resolution of PIV measurements: application to leaflet wake flow in a mechanical heart valve. Experiments in Fluids, 51(1), 161-176. doi:10.1007/s00348-010-1038-2. 
[15] Darwish, A., Di Labbio, G., Saleh, W., Smadi, O., \& Kadem, L. (2019). Experimental investigation of the flow downstream of a dysfunctional bileaflet mechanical aortic valve. Artificial Organs, 43(10). doi:10.1111/aor.13483.

[16] Mirvakili, N., Di Labbio, G., Saleh, W., \& Kadem, L. (2019). Flow characteristics in a model of a left ventricle in the presence of a dysfunctional mitral mechanical heart valve. Journal of Visualization, 23(1), 1-8. doi:10.1007/s12650-019-00611-3.

[17] Zolfaghari, H., \& Obrist, D. (2019). Absolute instability of impinging leading edge vortices in a submodel of a bileaflet mechanical heart valve. Physical Review Fluids, 4(12). doi:10.1103/physrevfluids.4.123901.

[18] Cheng, R., Lai, Y. G., \& Chandran, K. B. (2004). Three-dimensional fluid-structure interaction simulation of bileaflet mechanical heart valve flow dynamics. Annals of biomedical engineering, 32(11), 1471-1483. doi:10.1114/B:ABME.0000049032.51742.10.

[19] Domenichini, F., Pedrizzetti, G., \& Baccani, B. (2005). Three-dimensional filling flow into a model left ventricle. Journal of Fluid Mechanics, 539(-1), 179. doi:10.1017/s0022112005005550.

[20] Jain, K. (2020). Efficacy of the FDA nozzle benchmark and the lattice Boltzmann method for the analysis of biomedical flows in transitional regime. Medical \& Biological Engineering \& Computing, 58(8), 1817-1830. doi:10.1007/s11517-020-02188-8.

[21] Khellaf, B., \& Boussad, B. (2019). Computational hemodynamic investigation of a new bileaflet mechanical heart valve. SIMULATION, 96(5), 459-469. doi:10.1177/0037549719886364.

[22] Xu, X., Liu, T., Li, C., Zhu, L., \& Li, S. (2019). A Numerical Analysis of Pressure Pulsation Characteristics Induced by Unsteady Blood Flow in a Bileaflet Mechanical Heart Valve. Processes, 7(4), 232. doi:10.3390/pr7040232.

[23] Zbavitel, J., \& Fialová, S. (2019). A numerical study of hemodynamic effects on the bileaflet mechanical heart valve. EPJ Web of Conferences, 213, 02103. doi:10.1051/epjconf/201921302103.

[24] Redaelli, A., Bothorel, H., Votta, E., Soncini, M., Morbiducci, U., Del Gaudio, C., ... \& Grigioni, M. (2004). 3-D simulation of the St. Jude medical bileaflet valve opening process: fluid-structure interaction study and experimental validation. Journal of Heart Valve Disease, 13(5), 804-813.

[25] Fiedler, A. G., \& Tolis, G. (2018). Surgical Treatment of Valvular Heart Disease: Overview of Mechanical and Tissue Prostheses, Advantages, Disadvantages, and Implications for Clinical Use. Current Treatment Options in Cardiovascular Medicine, 20(1). doi:10.1007/s11936-018-0601-7.

[26] Voskoboinick, V., Kornev, N., \& Turnow, J. (2012). Study of Near Wall Coherent Flow Structures on Dimpled Surfaces Using Unsteady Pressure Measurements. Flow, Turbulence and Combustion, 90(4), 709-722. doi:10.1007/s10494-012-94339.

[27] Voskoboinick, V., Voskoboinick, A., Areshkovych, O., \& Voskoboinyk, O. (2016). Pressure fluctuations on the scour surface before prismatic pier. Scour and Erosion. doi:10.1201/9781315375045-115.

[28] Jun, B. H., Saikrishnan, N., \& Yoganathan, A. P. (2013). Micro Particle Image Velocimetry Measurements of Steady Diastolic Leakage Flow in the Hinge of a St. Jude Medical ${ }^{\circledR}$ Regent ${ }^{\mathrm{TM}}$ Mechanical Heart Valve. Annals of Biomedical Engineering, 42(3), 526-540. doi:10.1007/s10439-013-0919-y.

[29] Hedayat, M., \& Borazjani, I. (2019). Comparison of platelet activation through hinge vs bulk flow in bileaflet mechanical heart valves. Journal of Biomechanics, 83, 280-290. doi:10.1016/j.jbiomech.2018.12.003.

[30] Sacks, M. S., Schoen, F. J., \& Mayer, J. E. (2009). Bioengineering Challenges for Heart Valve Tissue Engineering. Annual Review of Biomedical Engineering, 11(1), 289-313. doi:10.1146/annurev-bioeng-061008-124903.

[31] Smood, B., Hara, H., Cleveland, D. C., \& Cooper, D. K. C. (2019). In Search of the Ideal Valve: Optimizing Genetic Modifications to Prevent Bioprosthetic Degeneration. The Annals of Thoracic Surgery, 108(2), 624-635. doi:10.1016/j.athoracsur.2019.01.054.

[32] Poli, D., Antonucci, E., Pengo, V., Migliaccio, L., Testa, S., Lodigiani, C., ... Palareti, G. (2018). Mechanical prosthetic heart valves: Quality of anticoagulation and thromboembolic risk. The observational multicenter PLECTRUM study. International Journal of Cardiology, 267, 68-73. doi:10.1016/j.ijcard.2018.04.042.

[33] H. Sadoon, and A. L. Bassam (2018). Incidence of Major Bleeding In Patients with Mechanical Heart Valve and Warfarin; a One Year Study. International Journal of Advanced Research, 6(2), 622-624. doi:10.21474/ijar01/6480.

[34] Li, W., Gao, Z., Jin, Z., \& Qian, J. (2020). Transient Study of Flow and Cavitation Inside a Bileaflet Mechanical Heart Valve. Applied Sciences, 10(7), 2548. doi:10.3390/app10072548.

[35] Kheradvar, A., \& Pedrizzetti, G. (2011). Vortex Formation in the Heart. Vortex Formation in the Cardiovascular System, 4579. doi:10.1007/978-1-4471-2288-3_3. 
[36] Ge, L., Dasi, L. P., Sotiropoulos, F., \& Yoganathan, A. P. (2007). Characterization of Hemodynamic Forces Induced by Mechanical Heart Valves: Reynolds vs. Viscous Stresses. Annals of Biomedical Engineering, 36(2), $276-297$. doi:10.1007/s10439-007-9411-x.

[37] Butchart, E. G. (2008). Antithrombotic management in patients with prosthetic valves: a comparison of American and European guidelines. Heart, 95(5), 430-436. doi:10.1136/hrt.2007.134726.

[38] A. Wilke, C. M. Wende, M. Horst, and D. Steverding, (2011). Thrombosis of A Prosthetic Mitral Valve After Withdrawal of Phenprocoumon Therapy. Cardiology Research. doi:10.4021/cr124w.

[39] Qian, J., Gao, Z., Hou, C., \& Jin, Z. (2019). A comprehensive review of cavitation in valves: mechanical heart valves and control valves. Bio-Design and Manufacturing, 2(2), 119-136. doi:10.1007/s42242-019-00040-z.

[40] Jin, Z., Qiu, C., Jiang, C., Wu, J., \& Qian, J. (2020). Effect of valve core shapes on cavitation flow through a sleeve regulating valve. Journal of Zhejiang University-SCIENCE A, 21(1), 1-14. doi:10.1631/jzus.a1900528.

[41] Lee, C. S., Chandran, K. B., \& Chen, L. D. (1996). Cavitation Dynamics of Medtronic Hall Mechanical Heart Valve Prosthesis: Fluid Squeezing Effect. Journal of Biomechanical Engineering, 118(1), 97-105. doi:10.1115/1.2795951.

[42] Voskoboinick, V. A., Grinchenko, V. T., \& Makarenkov, A. P. (2005). Pseudo-Sound behind an Obstacle on a Cylinder in Axial Flow. International Journal of Fluid Mechanics Research, 32(4), 488-510. doi:10.1615/interjfluidmechres.v32.i4.60.

[43] Williams, J. E. F. (1969). Hydrodynamic Noise. Annual Review of Fluid Mechanics, 1(1), 197-222. doi:10.1146/annurev.f1.01.010169.001213.

[44] Voskoboinick, V. A., Grinchenko, V. T., \& Makarenkov, A. P. (2003). Correlation Characteristics of a Wall Pressure Fluctuation Field in a Turbulent Boundary Layer Induced by a Longitudinal Flow along a Flexible Extended Cylinder. International Journal of Fluid Mechanics Research, 30(6), 644-650. doi:10.1615/interjfluidmechres.v30.i6.70.

[45] Voskoboinick, V., Voskoboinick, A., Voskoboinyk, O., \& Turick, V. (2020). Dimple Generators of Longitudinal Vortex Structures. Boundary Layer Flows - Theory, Applications and Numerical Methods. doi:10.5772/intechopen.83518.

[46] Voskoboinick, V. A., Turick, V. N., Voskoboinyk, O. A., Voskoboinick, A. V., \& Tereshchenko, I. A. (2018). Influence of the Deep Spherical Dimple on the Pressure Field under the Turbulent Boundary Layer. Advances in Computer Science for Engineering and Education, 23-32. doi:10.1007/978-3-319-91008-6_3.

[47] Voskoboinick, V. A., \& Makarenkov, A. P. (2004). Spectral Characteristics of the Pseudosonic Component of Hydrodynamical Noise In a Longitudinal Flow Around a Flexible Cylinder. International Journal of Fluid Mechanics Research, 31(1), 87-100. doi:10.1615/interjfluidmechres.v31.i1.70.

[48] Vinogradnyi, G. P., Voskoboinik, V. A., Grinchenko, V. T., \& Makarenkov, A. P. (1990). Spectral and correlation characteristics of the turbulent boundary layer on an extended flexible cylinder. Fluid Dynamics, 24(5), 695-700. doi:10.1007/bf01051721.

[49] Voskoboinick, V. A., Voskoboinick, A. V., Stepanovitch, V., Redaelli, A., Lucherini, F., Fiore, G. B., ... Chertov, O. (2019). Noise Of Open And Semi-Closed Bileaflet Prosthetic Mitral Valve. International Journal of Fluid Mechanics Research, 46(4), 337-348. doi:10.1615/interjfluidmechres.v46.i4.50.

[50] V. Voskoboinick, O. Voskoboinyk, and L. Tereshchenko, (2020) "Noise of bileaflet mechanical mitral valve in nonNewtonian fluid", Abstracts of the International scientific- practical conference "Theoretical Foundations of Modern Science and Practice", Melbourne, Australia, 117-122.

[51] Voskoboinick, V., Voskoboinick, A., Voskoboinick, A., Stepanovich, V., ... Hyzha, I. (2019). Vortex motion inside the hole of a complex geometry. Hydrodynamics and Acoustics, 1(3), 284-301. doi:10.15407/jha2018.03.284.

[52] Bendat, J. S., \& Piersol, A. G. (2011). Random data: analysis and measurement procedures (Vol. 729). John Wiley \& Sons.

[53] Voskoboinick, V. A., Redaelli, A., Chertov, O. R., Fiore, B., Voskoboinick, A. V., Rudnitckii, A. G., ... Lucherini, F. (2017). Effect of Fluid Viscosity on Noise of Bileaflet Prosthetic Heart Valve. Research Bulletin of the National Technical University of Ukraine "Kyiv Politechnic Institute", 0(5), 41-50. doi:10.20535/1810-0546.2017.5.112097. 